\title{
Age, Comorbid Conditions, and Racial Disparities in COVID-19 Outcomes
}

\author{
Zanthia Wiley $^{1}$ (D) - Julianne N. Kubes ${ }^{2} \cdot$ Jason Cobb $^{3} \cdot$ Jesse T. Jacob $^{1} \cdot$ Nicole Franks $^{4} \cdot$ Laura Plantinga $^{5} \cdot$ Janice Lea $^{3}$
}

Received: 2 October 2020 / Revised: 24 November 2020 / Accepted: 25 November 2020 / Published online: 7 January 2021

(C) W. Montague Cobb-NMA Health Institute 2021

\begin{abstract}
Background Black patients are disproportionately affected by COVID-19. The purpose of this study was to compare risks of hospitalization of Black and non-Black COVID-19 patients presenting to the emergency department and, of those hospitalized, to compare mortality and acute kidney injury.

Methods A retrospective cohort of 831 adult COVID-19 patients (68.5\% Black) who presented to the emergency departments of four academic hospitals, March 1, 2020-May 31, 2020. The primary outcome was risk of hospitalization among Blacks vs. nonBlacks. Secondary outcomes were mortality and acute kidney injury, among hospitalized patients.

Results The crude odds of hospitalization were not different in Black vs. non-Black patients; however, with adjustment for age, Blacks had 55\% higher odds of hospitalization. Mortality differed most in the model adjusted for age alone. Acute kidney injury was more common in the Black hospitalized patients, regardless of adjustment. Stratified analyses suggested that disparities in the risk of hospitalization and of in-hospital acute kidney injury were highest in the youngest patients.

Conclusions Our report shows that Black and non-Black patients presenting to the emergency department with COVID-19 had similar risks of hospitalization and, of those who were hospitalized, similar mortality when adjusted for multiple factors. Blacks had higher risk of acute kidney injury. Our results suggest that examination of disparities without exploration of the individual effects of age and comorbidities may mask important patterns. While stratified analyses suggest that disparities in outcomes may differ substantially by age and comorbid conditions, further exploration among these important subgroups is needed to better target interventions to reduce disparities in COVID-19 clinical outcomes.
\end{abstract}

Keywords COVID-19 $\cdot$ Disparities $\cdot$ Race $\cdot$ Comorbidities $\cdot$ Age $\cdot$ Acute kidney injury

\section{Introduction}

COVID-19 disproportionately affects Black patients [1-10]. Significant disparities in hospitalization and mortality among Black COVID-19 patients have been reported [1-5, 8]. Data from 6 hospitals in Atlanta found that $79 \%$ of hospitalized patients were Black and that Black race was independently associated with hospitalization [2]. In a large cohort of 3481 patients with COVID-19 in New Orleans, $70.4 \%$ of patients were Black, $76.9 \%$ of the hospitalized patients were Black,
Key Points Blacks and non-Blacks presenting to the emergency department with COVID-19 had similar risks of hospitalization and, of those hospitalized, similar mortality. Disparities in risk of hospitalization and of acute kidney injury were highest in younger patients.

\section{Zanthia Wiley}

zwiley@emory.edu

1 Division of Infectious Diseases - Emory University Hospital Midtown, Emory University School of Medicine, 550 Peachtree Street, 7th floor of Medical Office Tower, Atlanta, GA 30308, USA

2 Office of Quality and Risk, Emory Healthcare - Emory University Hospital Midtown, 550 Peachtree Street, Orr Building 8th floor, Atlanta, GA 30308, USA
3 Division of Renal Medicine - Emory University Hospital Midtown, Emory University School of Medicine, 550 Peachtree Street, 7th floor of Medical Office Tower, Nephrology Clinic, Atlanta, GA 30308, USA

4 Department of Emergency Medicine - Emory University Hospital Midtown, Emory University School of Medicine, 550 Peachtree Street, Orr Building 11th Floor, Atlanta, GA 30308, USA

5 Division of Geriatrics and Gerontology - WWHC, Emory University School of Medicine, 552, 1841 Clifton Road, Atlanta, GA 30329, USA 
and $70.6 \%$ of those who died were Black [4]. Although increased prevalence of comorbidities may contribute to higher risk of COVID-19 infection and hospitalization, social determinants of health, including employment as frontline and essential workers, economic instability, and higher numbers in households (e.g., multigenerational homes) also contribute to these disparities [2].

Characteristics and clinical outcomes of hospitalized patients with COVID-19 have been described [1-5]. However, few studies have examined in detail the extent to which clinical and other variables that may explain the consistently observed differences by race in outcomes. Identification of such factors would increase our understanding of the extent of racial disparities and what potential factors could be targeted to lessen these disparities. Additionally, this identification would help better risk-stratify COVID-19 patients at presentation and improve overall outcomes.

Leveraging electronic medical record (EMR) data from four Atlanta, GA, hospitals during the initial 3 months of the COVID-19 pandemic, we aimed to evaluate differences in hospitalization risk among adult Black and non-Black COVID-19 patients who present to the emergency department (ED). We also aimed to examine in-hospital acute kidney injury (AKI) and mortality by race, among patients hospitalized from the ED. For all three outcomes, we further examined the contributions of various patient characteristics to these associations.

\section{Methods}

\section{Study Population and Data}

A retrospective cohort of all COVID-19 patients who presented to the EDs of four academic hospitals in Atlanta, GA, between March 1, 2020, and May 31, 2020, was created. A COVID-19 patient was defined as an adult (age $\geq 18$ years old) with a positive molecular SARS-CoV-2 test from a nasopharyngeal, oropharyngeal, or lower respiratory tract specimen. One thousand sixty COVID-19 patients were identified in this time period; 144 patients were excluded due to missing race information. Of the remaining 916 patients, 831 patients who presented to the ED had complete demographic, clinical, and laboratory data from the time of presentation to the ED. Data on comorbid conditions were extracted from the clinical data warehouse, including encounters either as inpatient, ED, or outpatient clinic, from 1 year prior to the time of presentation to the $\mathrm{ED}$, including the $\mathrm{ED}$ encounter.

\section{Study Variables}

Race Race was self-reported in the EMR. For analyses, race was categorized as Black vs. non-Black (including white,
Asian, American Indian/Alaska Native, Native Hawaiian/ Pacific Islander, and multiple race).

Hospitalization The hospitalization outcome was defined as the first admission to the hospital from the ED. No patient in this cohort presented to the ED more than once.

In-hospital AKI AKI was defined among those hospitalized by Kidney Disease: Improving Global Outcomes (KDIGO) guidelines as $\geq 1.5$ times increase from baseline (first measured creatinine on hospitalization) at any point during the hospitalization or $\geq 0.3 \mathrm{mg} / \mathrm{dL}$ increase in serum creatinine within $48 \mathrm{~h}$ during the hospitalization [11].

In-hospital Mortality In-hospital mortality was defined among those hospitalized as a discharge status of "dead" from the hospitalization that started with the admission from the ED (yes/no), among those who were hospitalized from the ED.

Age Age in years at presentation to ED was categorized as 18 $49,50-64$, and $65+$.

Insurance Insurance at the time of ED presentation was categorized as Medicare, Medicaid, commercial, or uninsured.

Comorbid Conditions ICD-10 codes to define each comorbid condition were defined as in the Charlson Comorbidity Index (CCI); a CCI was also calculated for each patient based on administrative codes using previously described methods [12]. Comorbidities included were hypertension, diabetes, cardiovascular disease, chronic kidney disease (CKD), dementia, chronic pulmonary disease, end stage renal disease, cancer, and HIV/AIDs.

Estimated Glomerular Filtration Rate Laboratory values for serum creatinine and estimated glomerular filtration rate GFR (eGFR) at presentation were defined as the first result from time of arrival to the emergency department. The eGFR provided in the EMR was calculated using the Modification of Diet in Renal Disease (MDRD) equation [13].

\section{Statistical Analysis}

Patient characteristics were compared by race using chisquare or, when appropriate, Fisher's exact tests, for categorical variables and Wilcoxon rank sum tests for continuous variables. Univariable and multivariable logistic regression analyses were used to assess associations between race and outcomes. For multivariable models, an initial set of variables were chosen a priori to be included in the models based on clinical expertise and added sequentially; variables that were not associated with race or the outcome were not included in the final models. The included ED population served as the 
denominator for the hospitalization outcome; hospitalized patients served as the denominator for the AKI and mortality outcomes. To assess for potential effect modification in post hoc analyses, we ran stratified models by age (all three outcomes) and CKD (AKI outcome). R (version 3.6.3) was used for all analyses.

\section{Results}

\section{Characteristics of COVID-19 Patients Presenting to the ED, by Race}

Among 831 COVID-19 patients who presented to the ED, $68.5 \%$ (569) were Black and 3.9\% (32) were of Hispanic ethnicity (Table 1). $96.7 \%$ of the Black patients who presented to the ED had healthcare insurance compared to $93.9 \%$ of non-Black patients. Black patients who presented to the ED were younger; had a higher median serum creatinine at presentation; and a higher prevalence of obesity, diabetes, hypertension, dementia, HIV/AIDs, and CKD compared to nonBlack patients.

\section{Hospitalization Among COVID-19 Patients Presenting to the ED, by Race}

Black vs. non-Black COVID-19 patients presenting to the ED were more likely to be hospitalized from the ED $(82.4 \%$ vs $79.0 \%$ ) (Table 2). In crude models, the odds of being hospitalized were not statistically significant different in Black vs. non-Black patients; however, with adjustment for age, Black patients had 55\% higher odds to be hospitalized than nonBlack patients. Additional adjustment for insurance status rendered similar but non-statistically significant results; further adjustment for comorbid conditions and eGFR resulted in null associations (Table 2). In age-stratified crude models, Black COVID-19 patients who were 18-49 years old were more than twice as likely as their non-Black counterparts to be hospitalized from the ED $(\mathrm{OR}=2.47$ (95\% CI, 1.29-4.73)), whereas there was no association of race with this outcome among older age groups $(50-64,65+)$.

\section{Characteristics of COVID-19 Patients Admitted to the Hospital from the ED, by Race}

Of the 676 COVID-19 patients who were hospitalized from the ED, $69.4 \%$ were Black with only 1 patient identifying as Hispanic ethnicity (Table 1). Of the 207 non-Black patients admitted to the hospital, 7.2\% (15) were of Hispanic ethnicity. The percentage of patients with healthcare insurance was $98.1 \%$ in Black patients and $96.2 \%$ in non-Black patients. Obesity, diabetes, and CKD were more common in Blackhospitalized patients. Prevalence of hypertension and cardiovascular disease were similar in both Black and nonBlack patients ( 76.1 vs. 70.5 , and 34.3 vs. $35.3, p=0.15$ and 0.88 , respectively). Although the median serum creatinine at presentation was different between the hospitalized Black and non-Black cohorts $(1.2 \mathrm{mg} / \mathrm{dL}$ vs $1.1 \mathrm{mg} / \mathrm{dL}, p<0.0001)$, the median eGFR at presentation was similar $(64 \mathrm{~mL} / \mathrm{min}$ vs $65 \mathrm{~mL} / \mathrm{min}, p=0.26$ ).

\section{Acute Kidney Injury Among COVID-19 Patients Admitted to the Hospital from the ED, by Race}

AKI was more common in the Black-hospitalized patients compared to their non-Black counterparts (19.1\% vs $14.0 \%)$ (Table 3). In crude models, the odds of AKI were $44 \%$ higher in Black vs. non-Black COVID-19 patients; with adjustment for age and then insurance, Black patients had $67 \%$ and $64 \%$ higher odds of having AKI than non-Black patients. Additional adjustment for comorbid conditions and then eGFR showed 39\% and 37\% higher odds of AKI, although the associations were not statistically significant (Table 2). In age-stratified crude models, the magnitude of the association of Black vs. non-Black race with AKI was higher among younger age groups $(18-49, \mathrm{OR}=3.98(95 \% \mathrm{CI}, 0.50$ 31.7); 50-64, OR $=2.09$ (95\% CI, 0.66-8.8); and 65+, $\mathrm{OR}=1.42$ (95\% CI, 0.77-2.65)), but none of the associations were statistically significant. Associations of Black vs. nonBlack race with AKI were also higher, but not statistically significant, among those with $(\mathrm{OR}=1.81(95 \% \mathrm{CI}, 0.77-$ $4.56)$ ) vs. without ( $\mathrm{OR}=1.15$ (95\% CI, 0.62-2.22)) CKD.

\section{Mortality Among COVID-19 Patients Admitted to the Hospital from the ED, by Race}

In-hospital mortality was not significantly different between the Black and non-Black groups (11.9\% vs $10.9 \%$ ) Table 3 ). With adjustment of crude models for age and then insurance, Black patients had 54\% and 53\% higher odds of dying in the hospital than non-Black patients; additional adjustment for comorbid conditions and then eGFR showed $28 \%$ and $24 \%$ higher odds of death, although the associations were not statistically significant (Table 3). In age-stratified crude models, Black-hospitalized COVID-19 patients who were 50-64 years old were more than twice as likely as their non-Black counterparts to die (OR $=2.35$ (95\% CI, 0.29-19.3)), although not statistically significant; there was no association of race with mortality among younger (18-49) or older (65+) age groups.

\section{Discussion}

In our large academic health system, $82.4 \%$ of Black patients who presented to the ED were hospitalized compared to $76.7 \%$ for non-Black patients. On its own, this finding may 
Table 1 Baseline clinical characteristics, renal function, and underlying comorbidities of hospitalized Black, hospitalized non-Black, and nonhospitalized Black patients with COVID-19 presenting to the emergency department

\begin{tabular}{|c|c|c|c|c|c|c|c|}
\hline & All $(n=831)$ & $\begin{array}{l}\text { All Black } \\
(n=569)\end{array}$ & $\begin{array}{l}\text { All non-Black } \\
(n=262)\end{array}$ & $p$ value & $\begin{array}{l}\text { Hospitalized } \\
\text { Black }(n=469)\end{array}$ & $\begin{array}{l}\text { Hospitalized } \\
\text { non-Black }(n=207)\end{array}$ & $\mathrm{p}$ value \\
\hline \multicolumn{8}{|l|}{ Demographics } \\
\hline \multicolumn{8}{|l|}{ Age, $n(\%)$} \\
\hline $18-49$ & $218(26.2)$ & $569(27.4)$ & $62(23.7)$ & 0.29 & $109(23.2)$ & $30(14.5)$ & 0.01 \\
\hline $50-64$ & $249(30.0)$ & $193(33.9)$ & $56(21.4)$ & 0.0003 & $158(33.7)$ & $45(21.7)$ & 0.002 \\
\hline $65+$ & $364(43.8)$ & $220(38.7)$ & $144(55.0)$ & $<0.0001$ & $202(43.1)$ & $132(63.8)$ & $<0.0001$ \\
\hline \multicolumn{8}{|l|}{ Primary insurance, $n(\%)$} \\
\hline Medicare & $390(46.9)$ & $257(45.2)$ & $133(50.8)$ & 0.15 & $234(49.9)$ & $124(59.9)$ & 0.02 \\
\hline Medicaid & $38(4.6)$ & $29(5.1)$ & $9(3.4)$ & 0.38 & $23(4.9)$ & $8(3.9)$ & 0.69 \\
\hline Commercial & $368(44.2)$ & $264(46.4)$ & $104(39.7)$ & 0.08 & $203(43.3)$ & $67(32.4)$ & 0.01 \\
\hline Uninsured & $35(4.2)$ & $19(3.3)$ & $16(6.1)$ & 0.10 & $9(1.9)$ & $8(3.9)$ & 0.22 \\
\hline Male, $n(\%)$ & $419(50.4)$ & $284(49.9)$ & $135(51.5)$ & 0.72 & $240(51.1)$ & $107(51.7)$ & 0.97 \\
\hline Obese (BMI $\geq 30), n(\%)$ & $411(49.5)$ & $314(55.2)$ & $97(37.0)$ & $<0.0001$ & $270(57.6)$ & $78(37.7)$ & $<0.0001$ \\
\hline \multicolumn{8}{|l|}{ Race } \\
\hline Black & $569(68.5)$ & $569(100)$ & - & - & $469(100)$ & - & - \\
\hline White & $223(26.8)$ & - & $223(85.1)$ & - & - & $179(86.5)$ & - \\
\hline Asian & $32(3.9)$ & - & $32(12.2)$ & - & - & $24(11.6)$ & - \\
\hline American Indian/Alaska Native & $3(0.4)$ & - & $3(1.1)$ & - & - & $2(1.0)$ & - \\
\hline Native Hawaiian/Pacific Islander & $1(0.1)$ & - & $1(0.4)$ & - & - & $0(0.0)$ & - \\
\hline Multiple & $3(0.4)$ & - & $3(1.1)$ & - & - & $2(1.0)$ & - \\
\hline \multicolumn{8}{|l|}{ Ethnicity } \\
\hline Hispanic & $32(3.9)$ & $3(0.5)$ & $29(11.1)$ & $<0.0001$ & $1(0.2)$ & $15(7.2)$ & $<0.0001$ \\
\hline Non-Hispanic & $752(90.5)$ & $535(94.0)$ & $217(82.8)$ & $<0.0001$ & $444(94.7)$ & $181(87.4)$ & 0.002 \\
\hline Unknown & $47(5.7)$ & $31(5.4)$ & $16(6.1)$ & 0.83 & $24(5.1)$ & $11(5.3)$ & 0.99 \\
\hline \multicolumn{8}{|l|}{ Renal laboratory values } \\
\hline eGFR at presentation, median (IQR) & $69.0(51.5)$ & $69.0(56.5)$ & $69.0(43.3)$ & 0.46 & $64.0(59.0)$ & $65.0(40.0)$ & 0.36 \\
\hline $\mathrm{eGFR} \geq 60, n(\%)$ & $465(56.0)$ & $315(55.4)$ & $150(57.3)$ & 0.64 & $251(53.5)$ & $115(55.6)$ & 0.64 \\
\hline eGFR $30-59, n(\%)$ & $180(21.7)$ & $106(18.6)$ & $74(28.2)$ & 0.002 & $101(21.5)$ & $69(33.3)$ & 0.001 \\
\hline eGFR $15-29, n(\%)$ & $62(7.5)$ & $51(9.0)$ & $11(4.2)$ & 0.02 & $51(10.9)$ & $11(5.3)$ & 0.03 \\
\hline eGFR $<15, n(\%)$ & $80(9.6)$ & $67(11.8)$ & $13(5.0)$ & 0.003 & $66(14.1)$ & $11(5.3)$ & 0.002 \\
\hline $\begin{array}{l}\text { Serum creatinine at presentation, } \\
\text { median (IQR) }\end{array}$ & $1.1(0.7)$ & $1.2(1.0)$ & $1.0(0.5)$ & $<0.0001$ & $1.2(1.2)$ & $1.1(0.5)$ & $<0.0001$ \\
\hline \multicolumn{8}{|l|}{ Comorbidities, $n(\%)$} \\
\hline Charlson Comorbidity Index $>2$ & $298(35.9)$ & $218(39.3)$ & $80(30.5)$ & 0.04 & $212(45.2)$ & $77(37.2)$ & 0.06 \\
\hline Hypertension & $559(67.3)$ & $399(70.1)$ & $160(61.1)$ & 0.01 & $357(76.1)$ & $146(70.5)$ & 0.15 \\
\hline Diabetes & $301(36.2)$ & $227(39.9)$ & $74(28.2)$ & 0.002 & $212(45.2)$ & $66(31.9)$ & 0.002 \\
\hline Cardiovascular disease & $244(29.4)$ & $168(29.5)$ & $76(29.0)$ & 0.94 & $161(34.3)$ & $73(35.3)$ & 0.88 \\
\hline Dementia & $113(14.0)$ & $67(11.8)$ & $46(17.6)$ & 0.03 & $64(13.6)$ & $44(21.3)$ & 0.02 \\
\hline Chronic pulmonary disease & $155(18.7)$ & $106(18.6)$ & $49(18.7)$ & 0.99 & $92(19.6)$ & $40(19.3)$ & 0.99 \\
\hline Chronic kidney disease & $228(27.4)$ & $174(30.6)$ & $54(20.6)$ & 0.004 & $170(36.2)$ & $51(24.6)$ & 0.004 \\
\hline End stage renal disease & $70(8.4)$ & $62(10.9)$ & $8(3.1)$ & 0.0003 & $60(12.8)$ & $7(3.4)$ & 0.0003 \\
\hline Cancer & $59(7.1)$ & $43(7.6)$ & $16(6.1)$ & 0.54 & $42(9.0)$ & $15(7.2)$ & 0.56 \\
\hline HIV/AIDS & $15(1.8)$ & $15(2.6)$ & $0(0.0)$ & 0.02 & $13(2.8)$ & $0(0.0)$ & 0.03 \\
\hline
\end{tabular}

suggest that both Black and non-Black COVID-19 patients who are sick enough to seek care in the ED have a similar likelihood of being hospitalized. However, after adjustment for age, Black COVID-19 patients in the ED were 55\% more likely to be hospitalized, suggesting that, without taking racial differences in age distributions into account, we may miss disparities. In fact, our exploratory stratified analyses suggested that among the youngest patients there may be a 
Table 2 Hospitalization by race among COVID-19 patients presenting to the emergency department March 1, 2020-May 31,2020

\begin{tabular}{lll}
\hline & Black $(n=569)$ & Non-Black $(n=262)$ \\
\hline No. $(\%)$ of hospitalized patients & $469(82.4)$ & $207(79.0)$ \\
Odds ratio $(95 \%$ CI) & & \\
$\quad$ Unadjusted & $1.25(0.86-1.79)$ & $1.00(\mathrm{ref})$ \\
Adjusted for age & $1.55(1.04-2.29)$ & $1.00(\mathrm{ref})$ \\
Adjusted for age + insurance & $1.47(0.99-2.19)$ & $1.00(\mathrm{ref})$ \\
Adjusted for age + insurance + clinical & $1.11(0.73-1.69)$ & $1.00(\mathrm{ref})$ \\
Adjusted for age + insurance + clinical + presenting eGFR & $1.22(0.76-1.94)$ & $1.00(\mathrm{ref})$ \\
\hline
\end{tabular}

Clinical $=$ hypertension, diabetes, cardiovascular disease, chronic kidney disease, ESRD substantial disparity, with younger Black patients being more than twice as likely as similar non-Black patients to be hospitalized. The association of race with hospitalization was not attenuated by insurance status but was attenuated by adjustment for baseline comorbid conditions and renal function at presentation. While none of our models showed any significant associations with race and mortality which is consistent with other reports [1,2], when stratified by age, Blacks in the age group (50-64) had a twofold risk of dying, and thus, this particular age group may need particular attention in the future.

The odds of AKI was higher in Black- vs. non-Blackhospitalized COVID-19 patients; however, after adjustment for age and insurance, Black patients had even higher odds of AKI, $67 \%$ and $64 \%$, respectively, but further adjustments for comorbidities and eGFR rendered the association between race and AKI non-statistically significant. When crude models were stratified by age, the magnitude of the association of
Black vs. non-Black race with AKI was higher among younger age groups, although these associations did not achieve statistical significance. While the association of race and COVID-19-related AKI have been noted previously $[14,15]$ and speculated to be influenced by more severe underlying comorbidities in Blacks, our study does not confirm a significant relationship between race and comorbid factors and thus suggests there are other unmeasured factors that may play a role in these observed disparities. The pathophysiology of COVID-19-induced AKI is likely multifactorial and includes aggravation of preexisting conditions, acute inflammation, cardiorenal syndrome, hemodynamic instability, and hypovolemia [14-16]. Studies are ongoing to elucidate potential mechanisms and include examining the role of direct viral toxicity to the kidney and of genetic predispositions, such as APOL1 risk alleles [17].

While we seek to find the independent effect of race, it is worth considering that both the direct and indirect effects of
Table 3 Acute kidney injury and in-hospital mortality by race among COVID-19 patients hospitalized after presenting to the emergency department March 1, 2020-May 31, 2020

\begin{tabular}{|c|c|c|}
\hline \multicolumn{3}{|l|}{ Outcome } \\
\hline Acute kidney injury ${ }^{1}$ & Black $(n=409)$ & Non-Black $(n=200)$ \\
\hline No. of $(\%)$ patients with acute kidney injury & $78(19.1)$ & $28(14.0)$ \\
\hline \multicolumn{3}{|l|}{ Odds ratio for acute kidney injury $(95 \% \mathrm{CI})$} \\
\hline Unadjusted & $1.44(0.91-2.33)$ & 1.00 (ref) \\
\hline Adjusted for age & $1.67(1.04-2.75)$ & 1.00 (ref) \\
\hline Adjusted for age + insurance & $1.64(1.02-2.72)$ & 1.00 (ref) \\
\hline Adjusted for age + insurance + clinical $^{2}$ & $1.39(0.83-2.35)$ & 1.00 (ref) \\
\hline Adjusted for age + insurance + clinical $^{2}+$ presenting eGFR & $1.37(0.83-2.33)$ & 1.00 (ref) \\
\hline Mortality & Black $(n=469)$ & Non-Black $(n=207)$ \\
\hline No. $(\%)$ of patients who died & $56(11.9)$ & $27(10.3)$ \\
\hline \multicolumn{3}{|l|}{ Odds ratio for mortality $(95 \% \mathrm{CI})$} \\
\hline Unadjusted & $0.90(0.56-1.50)$ & 1.00 (ref) \\
\hline Adjusted for age & $1.54(0.91-2.68)$ & 1.00 (ref) \\
\hline Adjusted for age + insurance & $1.53(0.90-2.65)$ & 1.00 (ref) \\
\hline Adjusted for age + insurance + clinical $^{2}$ & $1.28(0.72-2.31)$ & 1.00 (ref) \\
\hline Adjusted for age + insurance + clinical $^{2}+$ presenting eGFR & $1.24(0.70-2.25)$ & 1.00 (ref) \\
\hline
\end{tabular}


race are important in describing disparities. Rather than being confounders of race and outcomes, the covariates we examined are likely on the causal pathway and thus mediators of the association [18]. Although we did not do a formal mediation analysis of direct and indirect effects, we can examine our sequentially adjusted models for a complete picture of racial disparity. For example, for all three outcomes, the addition of age increased the magnitude of the association of race with the outcome, which suggests that much of the effect of race is through the differing age distributions of Black vs. nonBlack COVID-19 patients presenting to the ED or being hospitalized. Insurance had little effect, likely related to the lack of difference in insured status we noted in our population. As others have reported, we found that comorbidities such as obesity, hypertension, diabetes, and CKD were more prevalent in Black patients; adjustment for these lessened the magnitude (and sometimes rendered null) the associations of race with outcomes. This suggests much of the effect we see after adjustment for age is due to the differing prevalence of comorbid conditions in Black vs. non-Black populations, which is likely due to sequelae of race and associated social exposures over the life course.

Although we found that hospitalized Black patients were $44 \%$ more likely to develop AKI, it is possible that the presentation eGFR reflected those with CKD and/or those with early signs of AKI upon presentation to the ED. We were not able to determine the evidence of prior CKD, by previous eGFRs, as these data were only available for $10 \%$ of the population, and thus is a limitation of our study. In addition, the use of ICD-10 billing codes for evidence of comorbidities, particularly for CKD, may have led to misclassification. For example, it is unknown whether the CKD ICD-10 codes were based on previous known renal function or if reflective of the eGFR upon presentation to the ED which may have in fact signaled the presence of AKI.

Other limitations deserve mention. It is unknown if patients presenting to the ED may have had outpatient encounters in the earlier stages of their COVID-19 illness, especially when testing was limited. And similarly, it is not known how (or if) short-term or long-term sequela may differ between race groups. There is the potential for selection bias, given that $14 \%$ of individuals had unknown race and were dropped from the analysis. There may be other confounders or mediators, such as markers of socioeconomic status beyond insurance (which did not vary in our population), that are unknown or unmeasured. In addition, our small Hispanic cohort does not allow for evaluation of disparate outcomes in this underserved population. Other limitations include that ORs overestimate risk in the setting of common outcomes and that our stratified analyses are limited in power.

While our study lacked more detailed socioeconomic factors (e.g., income, education history, frontline worker status), we did not observe that, as a representation of lack of or delayed access to healthcare, Black patients were less likely to be insured, as has been hypothesized. Conversely, 96.7\% of Black patients who presented to the ED and $98.1 \%$ of Black patients who were hospitalized had healthcare insurance (compared to $93.9 \%$ and $96.2 \%$ of non-Black patients, respectively); thus, our highly insured population could be viewed as a strength in that it allows for the examination of other putative risk factors beyond the lack of insurance coverage. Furthermore, since this report includes only patients who presented to the ED (and not outpatient clinics or urgent care centers), and insurance status might drive decisions to present to the ED and possibly be admitted to the hospital, it may have represented a sicker cohort, thus explaining the higher hospitalization risks for both racial groups, compared to other reports $[1,2]$.

Other strengths of our study include capturing a full 3 months of data during the initial peak of the COVID-19 pandemic in Georgia. We also include racial comparisons for all patients presenting to the ED as well as those hospitalized to better characterize the more at-risk cohort. In addition, our data analyses utilized a priori incremental modeling methods to better explain associations we observed with various exposures on the clinical outcomes. To our knowledge, this has not been fully detailed in other studies.

Our report highlights that in our highly insured cohort, Blacks and non-Blacks who present to the ED have similar risks of hospitalization and, of those who are hospitalized, similar in-hospital mortality when adjusted for multiple factors. We do find a disparity in risk for AKI in the Black cohort which needs to be further explored. However, our results also suggest that examination of disparities without exploration of the individual effects of age and comorbid conditions may mask important patterns. While our stratified analyses suggested that disparities in outcomes may differ substantially by age and comorbid conditions, further exploration among these important subgroups is needed to better target interventions to reduce disparities in clinical outcomes of COVID-19.

Author Contributions Each author contributed to the concept, design, and data interpretation of this study. J. N. K. performed data collection. Z. W. drafted the primary manuscript, and each author revised the manuscript critically for important intellectual content, and approved the final version of the manuscript.

Data Availability Data and materials are available.

\section{Compliance with Ethical Standards}

Conflict of Interest The authors declare that they have no conflicts of interest.

Consent to Participate The study was approved by the Emory University Institutional Review Board, which granted a waiver of consent and a waiver of Health Insurance Portability and Accountability Act authorization. The study was carried out in accordance with the principles embodies in the Declaration of Helsinki. 
Consent for Publication All authors consent for publication of this manuscript.

Code Availability $\mathrm{R}$ (version 3.6.3) was used for all analyses and is publicly available.

\section{References}

1. Gold JAW, Kong KK, Szablewski CM, et al. Characteristics and clinical outcomes of adult patients hospitalized with COVID-19Georgia, March 2020. MMWR Morb Mortal Wkly Rep. 2020;69(18):545-50. Published 2020 May 8. https://doi.org/10. 15585/mmwr.mm6918e1.

2. Killerby ME, Link-Gelles R, Haight SC, et al. Characteristics associated with hospitalization among patients with COVID-19-metropolitan Atlanta, Georgia, March-April 2020. MMWR Morb Mortal Wkly Rep. 2020;69(25):790-4. Published 2020 June 26. https:// doi.org/10.15585/mmwr.mm6925e1.

3. Azar KJM, Shen Z, Romanelli RJ, et al. Disparities in outcomes among COVID-19 patients in a Large Health Care System in California [published online ahead of print, 2020 May 21]. Health Aff (Millwood). 2020. https://doi.org/10.1377/hlthaff.2020.00598.

4. Price-Haywood EG, Burton J, Fort D, Seoane L. Hospitalization and mortality among black patients and white patients with COVID-19. N Engl J Med. 2020;382(26):2534-43. https://doi. org/10.1056/NEJMsa2011686.

5. Mahajan UM, Larkins-Pettigrew M. Racial demographics and COVID-19 confirmed cases and deaths: a correlational analysis of 2886 US counties [published online ahead of print, 2020 May 21]. J Public Health (Oxf). 2020:fdaa070. https://doi.org/10.1093/ pubmed/fdaa070.

6. Millett GA, Jones AT, Benkeser, et al. Assessing differential impacts of COVID-19 on black communities [published online ahead of print, 2020 May 14]. Ann Epidemiol. 2020;47:37-44. https://doi. org/10.1016/j.annepidem.2020.05.003.

7. Henning-Smith C, Tuttle M, Kozhimannil KB. Unequal distribution of COVID-19 risk among rural residents by race and ethnicity [published online ahead of print, 2020 May 12]. J Rural Health. 2020. https://doi.org/10.1111/jrh.12463.

8. Kim SJ, Bostwick W. Social Vulnerability and racial inequality in COVID-19 deaths in Chicago [published online ahead of print,
2020 May 21]. Health Educ Behav. 2020:1090198120929677513. https://doi.org/10.1177/1090198120929677.

9. Dorn AV, Cooney RE, Sabin ML. COVID-19 exacerbating inequalities in the US. Lancet. 2020;395(10232):1243-4. https:// doi.org/10.1016/S0140-6736(20)30893-X.

10. Hooper MW, Napoles AM, Perez-Stable E. National Institute on Minority Health and Health Disparities (NIMHD), National Institutes of Health, Bethesda, Maryland. JAMA Published online May 11. 2020:2466-7. https://doi.org/10.1001/jama.2020.8598.

11. Kidney Disease: Improving Global Outcomes (KDIGO) Acute Kidney Injury Work Group. KDIGO Clinical Practice Guideline for Acute Kidney Injury. Kidney Int Suppl. 2012;2:1-138.

12. Glasheen WP, Cordier T, Gumpina R, Haugh G, Davis J, Renda A. Charlson comorbidity index: $I C D-9$ update and $I C D-10$ translation. Am Health Drug Benefits. 2019;12(4):188-97.

13. Levey AS, Coresh J, Greene T, et al. Using standardized serum creatinine values in the modification of diet in renal study equation for estimating glomerular filtration rate. Ann Intern Med. 2008;145(4):247-54. https://doi.org/10.7326/0003-4819-145-4200608150-00004.

14. Hirsch JS, Ng JH, Ross DW, Sharma P, Shah HH, Barnett RL, et al. Acute kidney injury in patients hospitalized with COVID-19. Kidney Int. 2020;98(1):209-18. https://doi.org/10.1016/j.kint. 2020.05.006.

15. Mohamed MM, Lukitsch I, Torres-Ortiz AE, et al. Acute kidney injury associated with coronavirus disease 2019 in Urban New Orleans[published online ahead of print, 2020 May 13]. Kidney 360. 2020. https://doi.org/10.34067/KID.0002652020.

16. Ronco C, Bellomo R, Kellum JA. Acute kidney injury. Lancet. 2019;394:1949-64.

17. Larse CP, Bourne TD, Wilson JD, Saqqa O, Sharshir MA. Collapsing glomerulopathy in a patient with COVID-19. https:// doi.org/10.1016/j.ekir.2020.04.002.

18. VanderWeele TJ, Robinson WR. On causal interpretation of race in regressions adjusting for confounding and mediating variables. Epidemiology. 2014;25(4):472-84. https://doi.org/10.1097/EDE. 0000000000000105 .

Publisher's Note Springer Nature remains neutral with regard to jurisdictional claims in published maps and institutional affiliations. 